INTERNATIONAL JOURNAL OF MASS SPECTROMETRY 345-347: pp. 71-79. (2013)

http://dx.doi.org/10.1016/j.ijms.2012.08.031

Corresponding author: László Drahos (drahos.laszlo@ttk.mta.hu).

\title{
Fragmentation characteristics of glycopeptides
}

Károly Vékey, Oliver Ozohanics, Eszter Tóth, Anita Jekő, Ágnes Révész, Judit Krenyácz and László Drahos

Institute of Organic Chemistry, Research Centre for Natural Sciences, Hungarian Academy of Sciences, Budapest, Pusztaszeri ut 59-67, Hungary

\begin{abstract}
Mass spectrometric analysis of glycopeptides is an emerging strategy for analysis of glycosylation patterns. Here we present an approach using energy resolved collision induced decomposition (CID) spectra to determine structural features of glycopeptides. Fragmentation of multiply protonated glycopeptides proceeds by a series of competing charge separation processes by cleavage of a glycosidic bond, each producing two charged products: a singly charged, "B" type sugar (oxonium) ion, and a complementary high mass fragment. Energy requirements (activation energies) of these processes are similar to each other, and are far less, than that required for peptide fragmentation. At higher collision energies these first generation products fragment further, yielding a complex fragmentation pattern. Analysis of low energy spectra (those corresponding to ca. 50\% survival yield) are straightforward; the ions observed correspond to structural features present in the oligosaccharide, and are not complicated by consecutive reactions. This makes it feasible to identify and distinguish antenna- and core-fucosylated isomers; antenna fucosylation usually suggests presence of the Lewis- $X$ antigen. In general, analysis of the triply protonated molecules are most advantageous, where neutral losses and monosaccharide oxonium ion formation are less abundant.
\end{abstract}

*To whom correspondence should be addressed:

László Drahos

Research Centre for Natural Sciences, Hungarian Academy of Sciences

H-1525 Budapest, P.O. Box 17. Hungary

E-mail: drahos.laszlo@ttk.mta.hu

Phone: (36-1) 438-1100/536

Fax: (36-1) 438-1157 


\section{Highlights}

- We present energy resolved CID spectra and fragmentation characteristics of glycopeptides

- We suggest that glycopeptide analysis should be performed at low energy (SY50) to maximize information content

- Possibility to determine fucosylation site based on MS/MS

Running title: Fragmentation characteristics of glycopeptides

KEYWORDS: glycosylation; energy resolved mass spetra; fucosylation; alpha-1-glycoprotein (AGP); transferrin, cetuximab; 


\section{1) Introduction}

K.R. Jennings has been in the forefront of mass spectrometric research for several decades. In the 90's his interest turned to biological applications; including study of peptides and proteins. Beside research he took education seriously. I remember his excellent lectures and exercises on peptide fragmentation at a NATO conference in 2001 [1]. These were not only academically sound, but excellent teaching material as well. Although I had a fairly clear understanding of peptide fragmentation, I learnt a lot from these. He was kind enough to give me his overheads, which I still use in my own teaching. The present communication extends our knowledge of peptide fragmentation to glycopeptides. Following the steps of Keith I hope this will not only extend our understanding, but will also illustrate general concepts in a didactic manner.

Glycosylation is among the most important and common post-translational protein modifications (PTM) [2-4]. A given glycoprotein usually has a large number of glycoforms, having the same amino acid backbone, but differing in sugar composition. The set of glycoforms are often described as the glycosylation pattern. Glycoform analysis presents a significant challenge; mass spectrometry is one of the principal techniques for their analysis [5]. In order to study glycoforms, the first step nearly always is enzymatic digestion. Often the sugar chain is cleaved off the peptide backbone, resulting in an oligosaccharide mixture [6-9]. An alternative is to cleave the peptide chain using proteolytic enzymes (usually trypsin), which results in a peptide/glycopeptide mixture. Glycopeptides retain information on both the peptide and the corresponding oligosaccharide chains. This approach started to gain importance in recent years [8, 10-13], and presents two major advantages: (1) Glycosylation sites and also glycosylation patterns at each site can be determined. (2) Protein-specific glycosylation patterns can be determined not only for pure glycoprotein samples, but for glycoprotein mixtures as well. This simplifies sample preparation significantly, and may allow determining glycosylation patterns of specific glycoproteins in complex mixtures (like blood plasma) as well.

Glycopeptides are studied predominantly by mass spectrometry, and this approach has been reviewed recently $[5,11]$. When using electron capture or electron transfer dissociation (ECD, ETD), fragmentation of the peptide backbone is typical, yielding information on the peptide sequence and the site of glycosylation. Using collision induced decomposition (CID), fragmentation of the oligosaccharide chain takes place, yielding structural information on the oligosaccharide unit. Automatic analysis of glycopeptide mass spectra can be facilitated by theoretical spectra simulation [14]. A particularly important aspect of glycoprotein analysis is identification and quantitation of fucosylation, which may either take place on the antenna, or on the core GlcNAc unit. In a recent paper [15] energy resolved mass spectra were studied to confirm fucose-related fragmentation processes. This indicated that although fucose is lost easily from glycopeptides in $\mathrm{CID}$, but this process is insignificant in single stage mass spectra.

In the present paper we discuss energy resolved CID fragmentation processes of glycopeptides. This may help evaluating tandem mass spectra and to select optimum experimental conditions for glycopeptide analysis. Here we study $\mathrm{N}$-glycopeptides, although the presented concepts can be easily extended to O-glycopeptide fragmentation as well. 


\section{2) Methods and materials}

\subsection{Samples, chemicals and sample preparation}

Human alpha-1-acid glycoprotein (AGP) and serotransferin standards was purchased from SigmaAldrich (St. Louis, MO, USA). RapiGest SF (lyophilized sodium-3-[(2-methyl-2-undecyl-1,3-dioxolan4-yl)-methoxyl]-1-propane-sulfonate) was obtained from Waters (Milford, MA, USA). Cetuximab was obtained from Centocor BV, Hungary. Mass spectrometry grade trypsin (Promega Corporation, Madison, WI, USA) was used. All other reagents and enzymes were obtained from Sigma-Aldrich (St. Louis, MO, USA). Protein samples were digested by trypsin, according to protocols described recently [13]. The resulting peptide/glycopeptide mixtures were separated using a nanoflow UHPLC system (nanoAcquity UPLC, Waters, Milford, MA, USA) with reverse phase $\mathrm{C} 18$ column (BEH, $75 \mu \mathrm{m} \times 200 \mathrm{~mm}$ ) and a 110 min gradient [13].

\subsection{Mass spectrometry}

Compounds eluting from the nano-UHPLC were introduced into a high resolution QTOF Premier mass spectrometer (Waters, Milford, MA, USA), using electrospray ionization in positive ion mode. In order to avoid fragmentation in the ion source, the cone voltage was kept at a low voltage (15 V). Collision gas was argon, at $4 * 10^{-3} \mathrm{mbar}$; collision voltage was varied in the $0-50 \mathrm{~V}$ range to obtain energy resolved tandem mass spectra. To obtain good signal-to-noise ratio, MS/MS scan time was $2 \mathrm{~s}$, taking several spectra under identical conditions. To obtain energy dependent spectra of various charge states of the same compound, mutiple UHPLC runs were performed.

\subsection{Glycopeptide terminology}

Nomenclature used for complex oligosaccharides have been reported before $[11,13]$. Briefly, all glycoforms contain a pentasaccharide core $\left(\mathrm{GlCNAc}_{2} \mathrm{Man}_{3}\right)$ and a number of lactosamine (Gal-GlcNAc) antenna (2-4, labelled as Bi, Tri, Tetra). The antenna may be capped by a sialic acid unit, ' $S$ ' with the subsequent numeral indicates the number of sialic acid units present (e.g. BiS2). The oligosaccharides may contain a fucose unit, its presence is indicated by " $F$ ", like BiS2F1. The peptide sequence of the glycopeptide is indicated by the conventional one letter code, e.g. BiS2-NEEYNK.

\section{3) Results and Discussion}

Glycopeptides (like peptides) typically form multiply charged (protonated, occasionally cationized) ions under electrospray ionization conditions. The charge state of glycopeptides is somewhat lower than that of peptides of similar mass, so they are usually observed at higher $\mathrm{m} / \mathrm{z}$ ratios. The molecular mass of typical $\mathrm{N}$-glycopeptides is in the 3-4000 Da mass range; charge states $2+, 3+$ are commonly, 4+ occasionally observed. A major feature of glycopeptide fragmentation is, that these 
compounds require much less energy to fragment than peptides of similar mass to charge ratio. This is observed both in single stage [16], and in tandem mass spectra. Fig. 1 shows collision energy-dependent (energy resolved) CID spectra of an AGP (alpha-acid-glycoprotein) derived glycopeptide, $[\mathrm{BiS} 2 \cdot \mathrm{NEEYNK}+3 \mathrm{H}]^{3+}$. To observe a similar degree of fragmentation for a triply protonated peptide of the same mass would require approximately 3 times higher collision energy. The degree of fragmentation can be well described by the relative abundance of the molecular signal, which is defined as the survival yield [17], $S Y=M /(M+\Sigma F)$. Here $M$ indicates intensity of the molecular ion signal, $\Sigma \mathrm{F}$ the sum of the intensities of all fragment ions. "Fragmentation efficiency" is also a commonly used terminology; this defines the relative abundance of fragment ions $\left(F E=\Sigma F /\left(M+\sum F\right)=1-S Y\right)$. Fragmentation efficiency is also used with respect to a given fragment ion, in this case $\mathrm{FE}_{\mathrm{i}}=\mathrm{F}_{\mathrm{i}} /\left(\mathrm{M}+\sum \mathrm{F}_{\mathrm{i}}\right)$.

The spectra in Fig. 1 show relatively few fragmentation processes at low, and a large variety of fragments at high collision energy. Nearly all cleavages occur at the glycosidic bonds (i.e. between sugar residues; and correspond to $Y$ and $B$ type ions $[11,18]$. Fragmentation processes can be best evaluated at low collision voltage, around $10 \mathrm{~V}$ in the present case. (Note, $10 \mathrm{~V}$ collision voltage corresponds to $30 \mathrm{eV}$ laboratory frame collision energy for a 3+ ion.) The survival yield in this case is $60 \%$, the triply protonated molecule being the most abundant peak in the spectrum. The internal energy at this collision energy is relatively low, so consecutive fragmentation processes (described below) are absent, facilitating spectra evaluation. There is only one small peak in the spectrum which corresponds to neutral loss from the precursor ion (sialic acid loss at $\mathrm{m} / \mathrm{z} 904$ ). All other ions are formed by charge separation processes [19].

In the course of charge separation the triply protonated molecule breaks up into a singly charged and a doubly charged ions. Cleavage at a glycosidic bond forms a singly charged sugar (oxonium) ion and a doubly charged counterpart (easiest to describe as "sugar" or "antenna loss", Fig. 2). The doubly charged fragments are easy to identify, as they appear at a higher $\mathrm{m} / \mathrm{z}$ values than the parent triply protonated molecule. Three major fragmentation processes are observed for $[\mathrm{BiS2} \cdot \mathrm{NEEYNK}]^{3+}$ - these correspond to the loss of a singly charged sialic acid $\left([\mathrm{Sia}+\mathrm{H}]^{+}\right)$; singly charged whole 'antenna' ( [Sia.Gal.GlcNAc+H] ${ }^{+}$) and loss of a singly charged larger sugar, ( [Sia.Gal.GlcNAc.Man $+\mathrm{H}]^{+}$). With very low intensity loss (and formation) of a singly charged disaccharide, $\left([\mathrm{Sia} \cdot \mathrm{Gal}+\mathrm{H}]^{+}\right.$) can also be observed. Note, all of these fragments are produced by cleavage of a single bond (as indicated in Fig. 2), and represent structural units present in the starting compound. The 6 charged products of these three processes give rise to the major fragment ions in the $10 \mathrm{~V}$ spectrum shown in Fig. 1. Note, although the singly and doubly charged complementary fragments should have the same abundance, the observed signals have somewhat different abundances, due to differences in mass discrimination and detection efficiency, and due to possible secondary processes (at higher energies).

Increasing the collision energy complicates the spectra significantly. At $20 \mathrm{~V}$ (Fig. 1) the molecular species $\left([\mathrm{M}+3 \mathrm{H}]^{3+}\right)$ practically disappears, while the spectrum becomes dominated by consecutive processes. Easiest to identify among these are high mass singly charged ions: These are formed by losing a second 'antenna' from the doubly charged primary products. The fragmentation scheme in Fig. 2 shows these in simplified form; one such reaction cascade is given below:

$[\mathrm{M}+3 \mathrm{H}]^{3+} \rightarrow[\mathrm{Sia} \cdot \mathrm{Gal} \cdot \mathrm{GlcNAc}+\mathrm{H}]^{+}+[\mathrm{M}-\{\mathrm{Sia} \cdot \mathrm{Gal} \cdot \mathrm{GlcNAc}\}+2 \mathrm{H}]^{2+}$

$$
[\mathrm{M}-\{\mathrm{Sia} \cdot \mathrm{Gal} \cdot \mathrm{GlcNAC}\}+2 \mathrm{H}]^{2+} \rightarrow[\mathrm{Sia}+\mathrm{H}]^{+}+[\mathrm{M}-\{\mathrm{Sia} \cdot \mathrm{Gal} \cdot \mathrm{GlcNAc}\}-\mathrm{Sia}+\mathrm{H}]^{+}
$$


Note that the oxonium ion products overlap with those produced by direct cleavages. Larger singly charged oxonium ions may also fragment by cleavage of a glycosidic bond, e.g. [Sia.Gal.GlcNAc + $\mathrm{H}]^{+}$may yield $\left[\mathrm{Sia}+\mathrm{H}^{+}\right.$. Small neutral losses (like water loss from $[\mathrm{Sia}+\mathrm{H}]^{+}$) also occur. With some exceptions, most high energy fragments may be formed via several reaction channels, and therefore have limited utility for structure analysis.

One structurally important fragment observed at high collision energy (20 and $40 \mathrm{~V}$ spectra in Fig. 1 ) is the peptide chain with one sugar unit attached; observed as a singly charged ion ( [Pep-GlcNAc $+\mathrm{H}]^{+}$, which may also be described as the $\mathrm{Y} 1$ ion [11]). This has high relevance for structure analysis, as this ion clearly indicates the mass of the peptide chain (and therefore can be used to identify which glycoprotein gives rise to the studied glycopeptide) $[13,20]$. This ion may fragment further loosing the last GIcNAc unit, yielding a singly protonated peptide ion ( $[\mathrm{Pep}+\mathrm{H}]^{+}$ ). Another useful feature of high energy spectra are the abundant oxonium ions. Observing several oxonium ions in an MS/MS spectrum is clear indication that the studied compound contains an oligosaccharide chain (in a tryptic digest this clearly identifies a glycopeptide).

Energetics of fragmentation can be described in a quantitative manner using breakdown diagrams. These show the relative abundance of various fragments ( $F / M+\Sigma F)$ as a function of collision voltage. As described above, we use 'collision voltage' and not the more commonly used terminology 'collision energy' for two reasons: First, this clearly indicates the laboratory and not the center-of-mass frame of reference. Second, for multiply charges species the collision energy is obtained by multiplying the collision voltage and the charge state. Using 'collision voltage' makes it easier to relate results to experimental parameters, and avoids ambiguity. Note also, that the collision voltage, laboratory and center-of-mass collision energies are all linearly related.

The breakdown diagram of [BiS2.NEEYNK $]^{3+}$ is shown in Fig. 3a (see also the spectra in Fig. 1). For clarity only a few selected fragmentation processes are included. The complementary charge separation fragments of [Sia.Gal.GlcNAc $+\mathrm{H}]^{+}$and $[\mathrm{M}-\{\mathrm{Sia} \cdot \mathrm{Gal} \cdot \mathrm{GlcNAc}\}+2 \mathrm{H}]^{2+}$ ions and also neutral sialic acid loss are all formed at low collision voltage, and their intensity passes through a maximum around $15 \mathrm{~V}$. Other primary charge separation processes show similar energy dependence. Energy dependence of these processes is better illustrated in Fig. 3b, where the breakdown diagram is normalized to the respective ion abundances observed at $10 \mathrm{~V}$ collision voltage. Fig. $3 b$ clearly indicates similar energy dependence for all primary fragmentation processes at low collision voltages. This strongly suggests that the activation energies of these processes are also similar.

Above ca. $15 \mathrm{~V}$ most of these ions start to decompose, as indicated by decreasing intensities in Fig. $3 \mathrm{~b}$ (also evident in Fig. 3a). Doubly charged products fragment by charge separation reactions (as described above), while singly charges oligosaccharide oxonium ions fragment to yield smaller sugar fragments. The energy onset of these secondary processes can be well illustrated in Fig. 3c, in which case ion abundances are normalized to that observed at $30 \mathrm{~V}$ collision voltage.

The similar behavior of fragmentation efficiency curves shown in Fig. 3 suggests, that the cleavage of various glycosidic bonds requires similar activation energies. Both the formation and fragmentation of the large oligosaccharide oxonium ion [Sia.Gal.GlcNAc.Man $+\mathrm{H}]^{+}$at $\mathrm{m} / \mathrm{z} 819$ is somewhat easier than that of other oxonium ions, best illustrated in Fig. $3 \mathrm{~b}$. On the other hand 
cleavage between Gal and GIcNAc units on the antennae produces very small ion signals; suggesting that this reaction requires somewhat higher activation energy. Cleavage between Man and GICNAc on the oligosaccharide core has not been observed, suggesting that this reaction is unfavorable, either due to energetic or steric effects.

At this point it is interesting to compare these results to the energy resolved studies obtained by $\mathrm{Y}$. Wada [15]. In many respects the results are analogous, but there are some differences as well. First, results presented here show several abundant processes at low energy, and not only that of $[\mathrm{Sia} \cdot \mathrm{Gal} \cdot \mathrm{GlcNAc}+\mathrm{H}]^{+}$formation. Second, in our case not only the singly charged oxonium ions (i.e. $\mathrm{B}_{\mathrm{n}}$ ions), but the complementary high mass fragments (e.g. $[\mathrm{M}-\{\mathrm{Sia} \cdot \mathrm{Gal} \cdot \mathrm{GlcNAc}\}+2 \mathrm{H}]^{2+}$ ) fragments were also observed. Absence of these higher mass ions is likely due to discrimination effects in the ion trap instrument. Note that formation of a singly charged product from a triply charged ion must be accompanied by formation of a doubly charged ion; and such fragments were observed before [14].

Another important aspect of glycopeptide fragmentation is the influence of the charge state. This has been studied in a number of cases, and all showed analogous behavior. As example, Fig. 4 shows the survival yield curves of $2+, 3+$ and $4+$ charge states of a glycopeptide, TriS3.LVPVPITNATLDR (doubly, triply and quadruply protonated molecules, respectively). The Figure clearly shows that high charge states fragment at much lower voltages, than low charge states. Collision voltages corresponding to SY50 of the three charge states are 39, 17.5 and $8.5 \mathrm{~V}$, respectively. A major part of this difference is simply due to the fact, that the collision energy of an ion is directly proportional to the number of charges. In the present case the (laboratory frame) collision energies are 78, 52 and $34 \mathrm{eV}$, respectively. These values show that high charge states not only fragment at lower voltages, but also at lower collision energies. This in turn indicates, that the activation energy of fragmentation of high charge states is lower (or much lower) than those of low charge states. This is easily explained, as charge repulsion increases with the number of charges on the molecule; and as part of the charge repulsion is used to decrease the activation energy of charge separation processes [21]. As described above, glycopeptide fragmentation mainly proceeds by charge separation, so this effect decreases the activation energy of most processes.

Neutral losses are not affected by charge repulsion, so the activation energy of these processes is similar for all charge states. Spectra shown in Fig. 1 indicate that loss of a neutral sialic acid and the primary charge separation processes requires similar activation energy for the $3+$ ion. This should imply, that neutral losses should be more pronounced for $2+$ than for $3+$ or $4+$ ions. The mass spectra of doubly, triply and quadruply protonated TriS3.LVPVPITNATLDR (m/z 2135, 1424, and 1068) does indeed show this effect (Fig. 5): At a collision voltage corresponding to $50 \%$ survival yield of [TriS3.LVPVPITNATLDR $+2 \mathrm{H}]^{2+}$ neutral sialic acid loss ( $\left.\mathrm{m} / \mathrm{z} 1990\right)$ is one of the most abundant fragments; in the spectrum of the triply protonated compound it is a small, but clearly observable ion; while in the case of the quadruply protonated ion it cannot be observed. In other respects the fragmentation patterns of various protonated forms are analogous; and follow the pattern discussed in detail for the BiS2 glycoform (Figs. 1, 2 and 3). Not counting small neutral losses, fragmentation commences with a charge separation cleavage of a glycosidic bond. In the case of the doubly and triply protonated ion the most abundant is the [Sia.Gal.GlcNAc $+\mathrm{H}]^{+}(\mathrm{m} / \mathrm{z}$ 657). In the case of double protonated species the complementary $[\mathrm{M}-\{\text { Sia.Gal.GlcNAc }\}+\mathrm{H}]^{+}$is 
observed at $\mathrm{m} / \mathrm{z} 3615$ (shown in Fig. 5a). For the triply protonated compound the complementary $[\mathrm{M}-\{\text { Sia.Gal.GlcNAc }\}+2 \mathrm{H}]^{2+}$ product is a large peak (at $\mathrm{m} / \mathrm{z} 1808$ ). In the case of the quadruply protonated species both analogous products [Sia.Gal.GlcNAc $+\mathrm{H}]^{+}$and $[\mathrm{M}-\{\mathrm{Sia} \cdot \mathrm{Gal} \cdot \mathrm{GlcNAc}\}+$ $3 \mathrm{H}]^{3+}(\mathrm{m} / \mathrm{z}$ 1205) can be observed, but their intensity is significantly reduced. Another glycosidic cleavage leads to production of [Sia $+\mathrm{H}]^{+}(\mathrm{m} / \mathrm{z} 292)$ and complementary [M - Sia $\left.+\mathrm{nH}\right]^{\mathrm{n}+}$ ions. This leads to small ions in the case of double and triple protonation, but is the predominant process for the quadruply protonated molecule (Fig. $5 \mathrm{c}$ ). Differences in relative abundances of $[\mathrm{Sia}+\mathrm{H}]^{+}$and $[\mathrm{Sia} \cdot \mathrm{Gal} \cdot \mathrm{GlcNAc}+\mathrm{H}]^{+}$formation among different charge states are reasonable to explain based on charge repulsion. The most likely (energetically most favourable) protonation site is a GlcNAc unit, resulting in preferential cleavage beside GIcNAc and Man, leading to [Sia.Gal.GlcNAc $+\mathrm{H}]^{+}$ formation [15]. When there are many (four) charges (protons) in a molecule, charge repulsion shifts the most likely protonation site towards the end of the molecule, i.e. to the sialic acid units. This results in preferential $\left[\mathrm{Sia}+\mathrm{H}^{+}\left(\right.\right.$and $[\mathrm{M}-\mathrm{Sia}+3 \mathrm{H}]^{3+}$ ) formation in the quadruply protonated species. The third type of glycosidic bond cleavage observed for BiS2 glycoform, cleavage between the core mannose units, are also found in TriS3. However, due to sensitivity reasons, the products were observed only for the triply protonated ions. For the doubly charged ions neutral losses, for the $4+$ species preferential $[\mathrm{Sia}+\mathrm{H}]^{+}$formation dominates, as discussed above. Increasing the collision energy consecutive reactions start to appear, just as in the case shown in Fig. 1 . These eventually lead to singly charged mono and oligosaccharides (oxonium ions) and to the [Pep + $\mathrm{GlcNAc}^{+}$and $[\mathrm{Pep}]^{+}$fragments. Fragmentation of other glycopeptides follows the same rules as described above. The peptide chain influences fragmentation only to a minor degree; collision energy dependence and fragmentation of the oligosaccharide chain is closely analogous to that discussed for the BiS2 and TriS3 glycoforms discussed in detail above.

Fucose containing glycopeptides have been studied by Y. Wada [15], indicating that (a) these compounds preferentially loose fucose in CID but (b) can be quantitated under low energy conditions (in which case there is no fragmentation). These findings are analogous to our energy resolved studies on sialylated glycoforms [16]. Energy-resolved fragmentation characteristics of glycopeptides, as discussed above, can be used to distinguish (identify) antenna $(\alpha 1,3 / 4)$ and core $(\alpha 1,6)$ fucosylated glycoforms. Fig. $6 a$ and b shows the low energy (SY50) spectra of triply protonated BiS2F1-NEEYNK and TriS3F1-LVPVPITNATLDR glycopeptides; where annotation of the major components are also indicated. These are fucosylated analogs of the BiS2 and TriS3 glycoforms shown in Fig. $1 \mathrm{~b}$ and $5 \mathrm{~b}$; fragmentation processes are analogous, even ion abundances are similar. Both glycoforms are derived from human AGP; fucose is known to be located on the antenna. The presence of fucose opens up the possibility of neutral fucose loss [15], which yields ions of similar abundance to that of neutral sialic acid loss. In the $3+$ species both are small peaks (ca. 5\% of the total fragment ion abundance); analytically these are not useful. Structurally most indicative are the peaks due to primary charge separation process leading to [Sia.Gal.GlcNAc.Fuc + $\mathrm{H}]^{+}$and $[\mathrm{M}-\{\mathrm{Sia} \cdot \mathrm{Gal} \cdot \mathrm{GlcNAc} \cdot \mathrm{Fuc}\}+2 \mathrm{H}]^{2+}$ (loss of a fucosylated antenna). Presence of these peaks can only be explained by fucosylation on the antenna; observing both peaks provides further confirmation. Both peaks are abundant, their intensity is somewhat smaller than those of the $[\text { Sia.Gal.GlcNAc.Fuc }+\mathrm{H}]^{+}$and $[\mathrm{M}-\{\mathrm{Sia} \cdot \mathrm{Gal} \cdot \mathrm{GlcNAc} \cdot \mathrm{Fuc}\}+2 \mathrm{H}]^{2+}$ (which structural units are also present in the compound). A medium and high energy the spectra show the [Pep.GlcNAc] ${ }^{+}$ion; but no signals are observed due to [Pep.GlcNAc.Fuc] ${ }^{+}$; confirming that there is no core-fucosylation (spectra are not shown). 
Core fucosylation, on the other hand, is evident in the spectrum of the BiS2F1.QQQHLFGSNVTDCSGNFCLFR glycoform (derived from human transferrin). The spectrum in insert of Fig. $6 \mathrm{c}$ shows both [Pep.GIcNAc $+2 \mathrm{H}]^{2+}$ and [Pep.GlcNAc.Fuc $\left.+2 \mathrm{H}\right]^{2+}$ ions in approximately 1:1 ratio; indicating fucosylation on the core GlcNAc unit. The low mass primary fragment $[\mathrm{Sia} \cdot \mathrm{Gal} \cdot \mathrm{GlcNAc}+\mathrm{H}]^{+}$is a major peak in the spectrum, while only traces of [Sia.Gal.GlcNAc.Fuc + $\mathrm{H}]^{+}$are observed (signal intensity is only $3 \%$ that of [Sia.Gal.GlcNAc $\left.+\mathrm{H}\right]^{+}$). This suggests that antenna fucosylation is present in very small amounts in this ion. Comparing signal intensities in Fig. $6 a \mathrm{~b}$ and $\mathrm{c}$ suggests that BiS2F1-QQQHLFGSNVTDCSGNFCLFR contains ca. $5-10 \%$ antenna and 90-95\% core fucosylation. The last example is GlcNAc $_{4}$ Man $_{3} \cdot$ EEQYNSTYR glycopeptide, derived a pharmaceutical antibody, Cetuximab. This contains two truncated antennas, the low energy spectrum of the doubly protonated glycopeptide is shown in Fig. 6d. Fragmentation is analogous to that of other glycopeptides discussed above. The largest fragment peak in the spectrum corresponds to a charge separation process leading to 'antenna loss' - as the compound contains a truncated antenna, this is a monosaccharide unit, [GlcNAc $+\mathrm{H}]^{+}$. The corresponding complementer ion, $[\mathrm{M}-\mathrm{GlcNAc}+\mathrm{H}]^{+}$is also visible at $\mathrm{m} / \mathrm{z} 2431$ as a low intensity peak. Traces of the fucosylated antenna [GICNAc.Fuc $+\mathrm{H}]^{+}$can also be observed, but abundance of this peak is only $5 \%$ that of [GICNAc $+\mathrm{H}]^{+}$. On the other hand, both [Pep.GlcNAc] ${ }^{+}$and $[\text {Pep.GlcNAc.Fuc }]^{+}$ions are observed; their abundance ration is approximately 1:1 ratio, like in the case of transferrin (Fig. $6 c)$. This indicates, that Cetuximab is predominantly core fucosylated $[22,23]$; antenna fucosylation is present in very small (very approximately 10\%) amount.

\section{4) Conclusions}

Energy-resolved CID spectra of glycopeptides show a number of important features. This information on fragmentation mechanism can be well utilized for practical applications and for glycoprotein analysis:

1) Fragmentation starts with competing cleavages of glycosidic bonds. These are charge separation processes, each yielding a singly charged B-type sugar fragment (oxonium ion) and a complementary high mass product. Activation energies of these alternative cleavages are close to each other, as shown by their similar increase at low collision voltage (Fig. 3b). Activation energies decrease with increasing charge state, and are much lower than those corresponding to peptide fragmentation. Optimum collision voltage for glycopeptides is 3-5 times lower than that typically used for peptides of the same mass and charge state.

2) Second generation products are also formed by glycosidic bond cleavages; the collision voltage necessary to induce these processes is approximately two times higher than that needed for the first cleavage process (compare Fig. $3 b$ and $c$ ). These secondary processes create a multitude of fragments and alternative reaction sequences and, in practice, are difficult to evaluate. An important secondary process, however, is formation of the [Pep.GlcNAc] ${ }^{+}$fragment (the $Y_{1}$ ion).

3) Neutral monosaccharide losses (B type cleavages, but with proton transfer to yield a small neutral sugar fragment) often take place from multiply protonated molecules. Sugar losses take place only from the ends of the oligosaccharide chains - i.e. mostly silalic acid or fucose (if present in the molecule). Glycopeptides with truncated antenna may loose the 
monosaccharide from the end of the chain (e.g. neutral GlcNAc loss from GICNAc $_{4} M_{3} \cdot n_{3}$ EEQYNSTYR peptide of Cetuximab, Fig. $6 \mathrm{~d}$ ). These processes are abundant only in lower charge states (doubly protonated molecules), and do not provide much structural information.

4) Spectrum analysis at low collision voltage, that corresponding to about $50 \%$ survival yield, provides important advantages. This is below the onset of consecutive reactions (Fig. 3); so spectra are easier to evaluate, and fragments can be directly related to the structure of the oligosaccharide. In most cases fragmentation of triply protonated molecules give best results; cleavage of the antenna between GlcNAc and Man is usually the most abundant process. Higher charge states are dominated by sialic acid formation; while in low charge states neutral losses predominate.

5) The fucosylation site can be determined from fragmentation. Antenna fucosylation in most human proteins corresponds to the presence of immunologically important Lewis- $X$ (or sialylated Lewis X) antigen. Presence or absence of antenna fucosylation is clearly indicated in the low energy spectrum by observing the singly charged antenna - [Sia.Gal.GlcNAc $+\mathrm{H}]^{+}$ at $\mathrm{m} / \mathrm{z} 657$ and [Sia.Gal.GlcNAc.Fuc $+\mathrm{H}]^{+}$at $\mathrm{m} / \mathrm{z}$ 803. In antenna fucosylated glycoform the abundance ratio of $\mathrm{m} / \mathrm{z} 803 / 657$ is approximately $30-50 \%$ (Fig. $6 a$ and b, but observed in several other cases as well). In the case of de-sialylated compounds or compounds with truncated antenna, analogous ion ratios may be observed. Core fucosylation is indicated by the presence of [Pep.GIcNAc.Fuc] $]^{+}$; which has a similar abundance to that of [Pep.GIcNAc] ${ }^{+}$.

6) High energy spectra, obtained at 2-4 times higher voltage than that corresponding to $50 \%$ survival yield are often useful to identify the [Pep.GlcNAc] fragment, and therefore the mass of the peptide chain. Such high energy spectra are also useful to identify low mass oxonium ions, which can be identified reliably even if the signal to noise ratio of the tandem mass spectrum is very low. This can be used to confirm, that the studied ion corresponds to a glycopeptide.

\section{5) Acknowledgements}

This work has been supported by the Hungarian Scientific Research fund (grant No. OTKA NK83857) and National Office for Research Technology (KMOP-1.1.2-07/1-2008-0002).

\section{6) References}

[1] K.R. Jennings, in: NATO-ASI on "Mass Spectrometry in Biomolecular Sciences", Tetouan, Morocco 2001.

[2] A. Varki, The essentials of glycobiology, in, Cold Spring Harbor Laboratory Press, Spring Harbor, 1999. 
[3] S.A. Brooks, M.V. Dwek, S. U., Functional \& Molecular Glycobiology, Bios scientific publishers LTD, 2002.

[4] H.R. Morris, S. Chalabi, M. Panico, M. Sutton-Smith, G.F. Clark, D. Goldberg, A. Dell, Glycoproteomics: Past, present and future, International Journal of Mass Spectrometry, 259 (2007) 16-31.

[5] H.J. An, C.B. Lebrilla, Structure Elucidation of Native N- and O-Linked Glycans by Tandem Mass Spectrometry (Tutorial), Mass Spectrometry Reviews, 30 (2011) 560-578.

[6] O. Gornik, L. Royle, D.J. Harvey, C.M. Radcliffe, R. Saldova, R.A. Dwek, P. Rudd, G. Lauc, Changes of Serum Glycans During Sepsis and Acute Pancreatitis, Glycobiology, 17 (2007) 1321-1332.

[7] K. Marino, J. Bones, J.J. Kattla, P.M. Rudd, A systematic approach to protein glycosylation analysis: a path through the maze, Nature Chemical Biology, 6 (2010) 713-723.

[8] T. Imre, G. Schlosser, G. Pocsfalvi, R. Siciliano, E. Molnar-Szollosi, T. Kremmer, A. Malorni, K. Vekey, Glycosylation site analysis of human alpha-1-acid glycoprotein (AGP) by capillary liquid chromatography-electrospray mass spectrometry, J. Mass Spectrom., 40 (2005) 14721483.

[9] T. Imre, T. Kremmer, K. Heberger, E. Molnar-Szollosi, K. Ludanyi, G. Pocsfalvi, A. Malorni, L. Drahos, K. Vekey, Mass spectrometric and linear discriminant analysis of N-glycans of human serum alpha-1-acid glycoprotein in cancer patients and healthy individuals, Journal of Proteomics, 71 (2008) 186-197.

[10] H. Wang, C.H. Wong, A. Chin, A. Taguchi, A. Taylor, S. Hanash, S. Sekiya, H. Takahashi, M. Murase, S. Kajihara, S. Iwamoto, K. Tanaka, Integrated mass spectrometry-based analysis of plasma glycoproteins and their glycan modifications, Nature Protocols, 6 (2011).

[11] E.D. Dodds, Gas-phase dissociation of glycosylated peptide ions, Mass Spectrometry Reviews, (2012) n/a-n/a.

[12] H. Narimatsu, H. Sawaki, A. Kuno, H. Kaji, H. Ito, Y. Ikehara, A strategy for discovery of cancer glyco-biomarkers in serum using newly developed technologies for glycoproteomics, Febs Journal, 277 (2010) 95-105.

[13] O. Ozohanics, L. Turiák, A. Puerta, K. Vékey, L. Drahos, High-performance liquid chromatography coupled to mass spectrometry methodology for analyzing site-specific $\mathrm{N}$ glycosylation patterns, Journal of Chromatography A.

[14] Z. Zhang, B. Shah, Prediction of Collision-Induced Dissociation Spectra of Common NGlycopeptides for Glycoform Identification, Analytical Chemistry, 82 (2010) 10194-10202.

[15] M. Tajiri, M. Kadoya, Y. Wada, Dissociation Profile of Protonated Fucosyl Glycopeptides and Quantitation of Fucosylation Levels of Glycoproteins by Mass Spectrometry, Journal of Proteome Research, 8 (2008) 688-693.

[16] J. Krenyacz, L. Drahos, K. Vekey, Collision energy and cone voltage optimisation for glycopeptide analysis, European Journal of Mass Spectrometry, 15 (2009) 361-365.

[17] C. Collette, L. Drahos, E.D. Pauw, K. Vékey, Comparison of the Internal Energy Distributions of ions Produced by Different Electrospray Sources, Rapid Communications in Mass Spectrometry, 12 (1998) 1673-1678.

[18] B. Domon, C.E. Costello, A systematic nomenclature for carbohydrate fragmentations in FABMS/MS spectra of glycoconjugates, Glycoconjugate Journal, 5 (1988) 397-409.

[19] R.G. Cooks, J.H. Beynon, R.M. Caprioli, G.R. Lester, Metastable Ions, Elsevier, Amsterdam, 1973. 
[20] O. Ozohanics, J. Krenyacz, K. Ludanyi, F. Pollreisz, K. Vekey, L. Drahos, GlycoMiner: a new software tool to elucidate glycopeptide composition, Rapid Communications in Mass Spectrometry, 22 (2008) 3245-3254.

[21] K. Vekey, G. Pocsfalvi, CALCULATION OF THE KINETIC-ENERGY RELEASE OF CHARGE SEPARATION PROCESSES, Organic Mass Spectrometry, 27 (1992) 1203-1209.

[22] C. Wacker, C.N. Berger, P. Girard, R. Meier, Glycosylation profiles of therapeutic antibody pharmaceuticals, European Journal of Pharmaceutics and Biopharmaceutics, 79 (2011) 503 507.

[23] J. Qian, T. Liu, L. Yang, A. Daus, R. Crowley, Q.W. Zhou, Structural characterization of N-linked oligosaccharides on monoclonal antibody cetuximab by the combination of orthogonal matrix-assisted laser desorption/ionization hybrid quadrupole-quadrupole time-of-flight tandem mass spectrometry and sequential enzymatic digestion, Anal. Biochem., 364 (2007) 8-18. 


\section{7) Figure Captions}

Fig. 1. Energy resolved tandem mass spectra of the triply protonated [BiS2-NEEYNK $]^{3+}$ glycopeptide derived from AGP. Spectra were obtained at 0, 10, 20 and $40 \mathrm{~V}$ collision voltage. The spectrum at low voltage shows mainly direct fragmentation of the triply protonated molecule, while at higher energies products of consecutive reactions dominate the spectra. See text for details.

Fig. 2. Fragmentation scheme of BiS2 type glycopeptides. All primary, and a few selected consecutive reaction processes are shown. Fragmentation of glycopeptides with different sugar chains is analogous.

Fig.3. Breakdown diagrams of $[\mathrm{BiS2} \cdot \mathrm{NEEYNK}]^{3+}$ showing selected fragmentation processes. (a) Conventional format, showing fragmentation processes and the survival yield curve in different scales. (b) Breakdown diagram showing some first generation product ions, renormalized to intensity observed at $10 \mathrm{~V}$ collision voltage. This shows that at low energy intensity of all these processes increase with collision voltage at a similar rate. At higher voltages secondary processes change intensities at a different rate. (c) Breakdown diagram showing selected second generation fragments. This shows that these processes have approximately similar onset energies.

Fig. 4. Survival yield curves of the TriS3.LVPVPITNATLDR glycopeptide; observed for the $2+, 3+$ and $4+$ charge states (2, 3 and 4-times protonated forms).

Fig. 5. Tandem mass spectra of doubly, triply and quadruply protonated TriS3.LVPVPITNATLDR glycopeptide, derived from AGP. All spectra correspond to ca. $50 \%$ survival yield; and have been taken at $35 \mathrm{~V}, 15 \mathrm{~V}$ and $7 \mathrm{~V}$ collision voltage. Fragmentation is discussed in detail in the text.

Fig. 6. Low energy CID spectra of selected glycopeptides, containing a fucose residue. See text for $\begin{array}{lllll}\text { discussion. } & \text { (a) TriS3F1.LVPVPITNATLDR, } & \text { (b) BiS2F1.NEEYNK, } & \text { (c) }\end{array}$ BiS2F1·QQQHLFGSNVTDCSGNFCLFR, (d) N4H3SOF1·EEQYNSTYR 
Fig. 1. Energy resolved tandem mass spectra of the triply protonated [BiS2-NEEYNK $]^{3+}$ glycopeptide derived from AGP. Spectra were obtained at 0,10, 20 and $40 \mathrm{~V}$ collision voltage. The spectrum at low voltage shows mainly direct fragmentation of the triply protonated molecule, while at higher energies products of consecutive reactions dominate the spectra. See text for details.

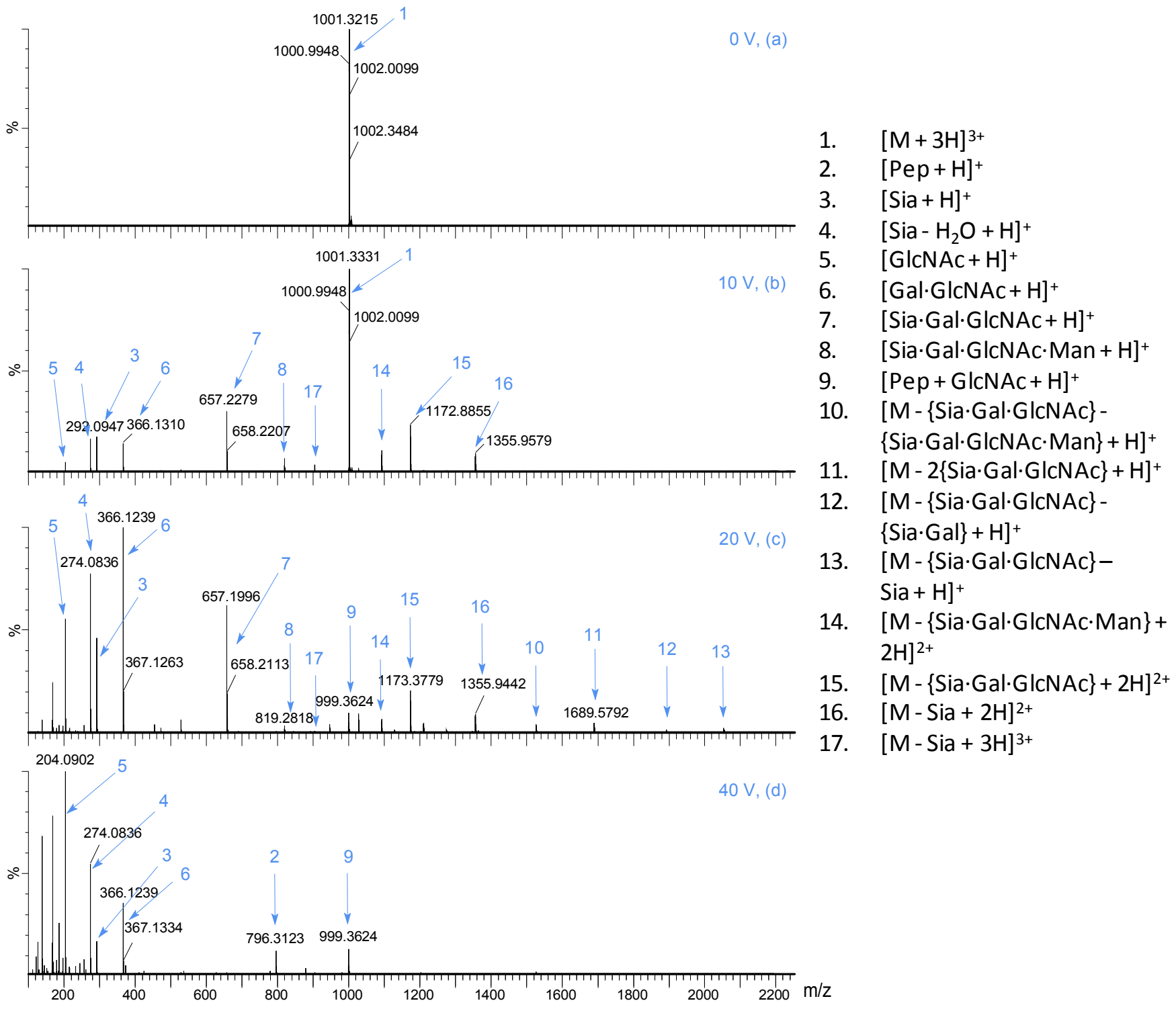


Fig. 2. Fragmentation scheme of BiS2 type glycopeptides. All primary, and a few selected consecutive reaction processes are shown. Fragmentation of glycopeptides with different sugar chains is analogous.

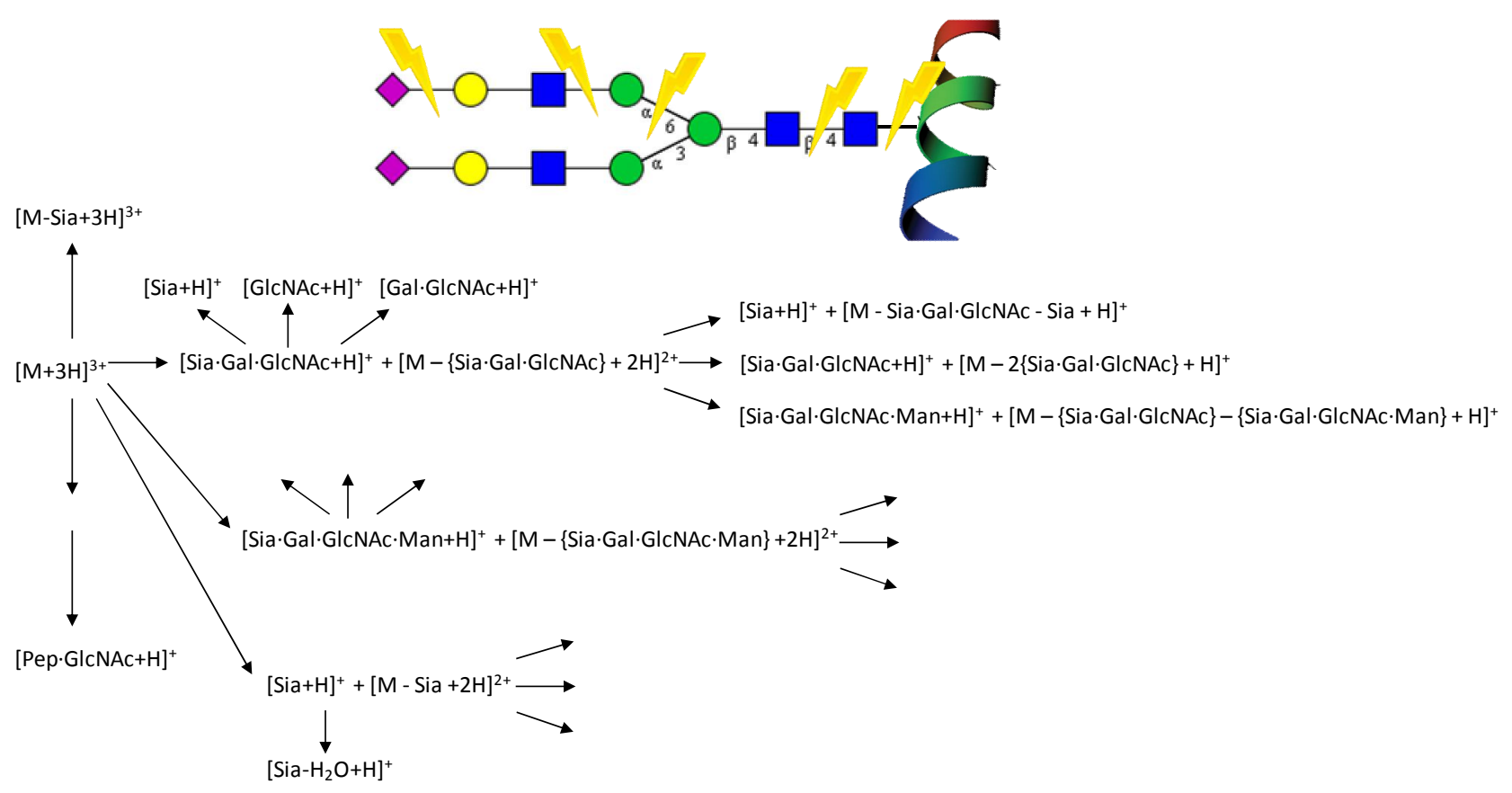


Fig.3. Breakdown diagrams of $[\mathrm{BiS2} \cdot \mathrm{NEEYNK}]^{3+}$ showing selected fragmentation processes. (a) Conventional format, showing fragmentation processes and the survival yield curve in different scales. (b) Breakdown diagram showing some first generation product ions, renormalized to intensity observed at $10 \mathrm{~V}$ collision voltage. This shows that at low energy intensity of all these processes increase with collision voltage at a similar rate. At higher voltages secondary processes change intensities at a different rate. (c) Breakdown diagram showing selected second generation fragments. This shows that these processes have approximately similar onset energies.
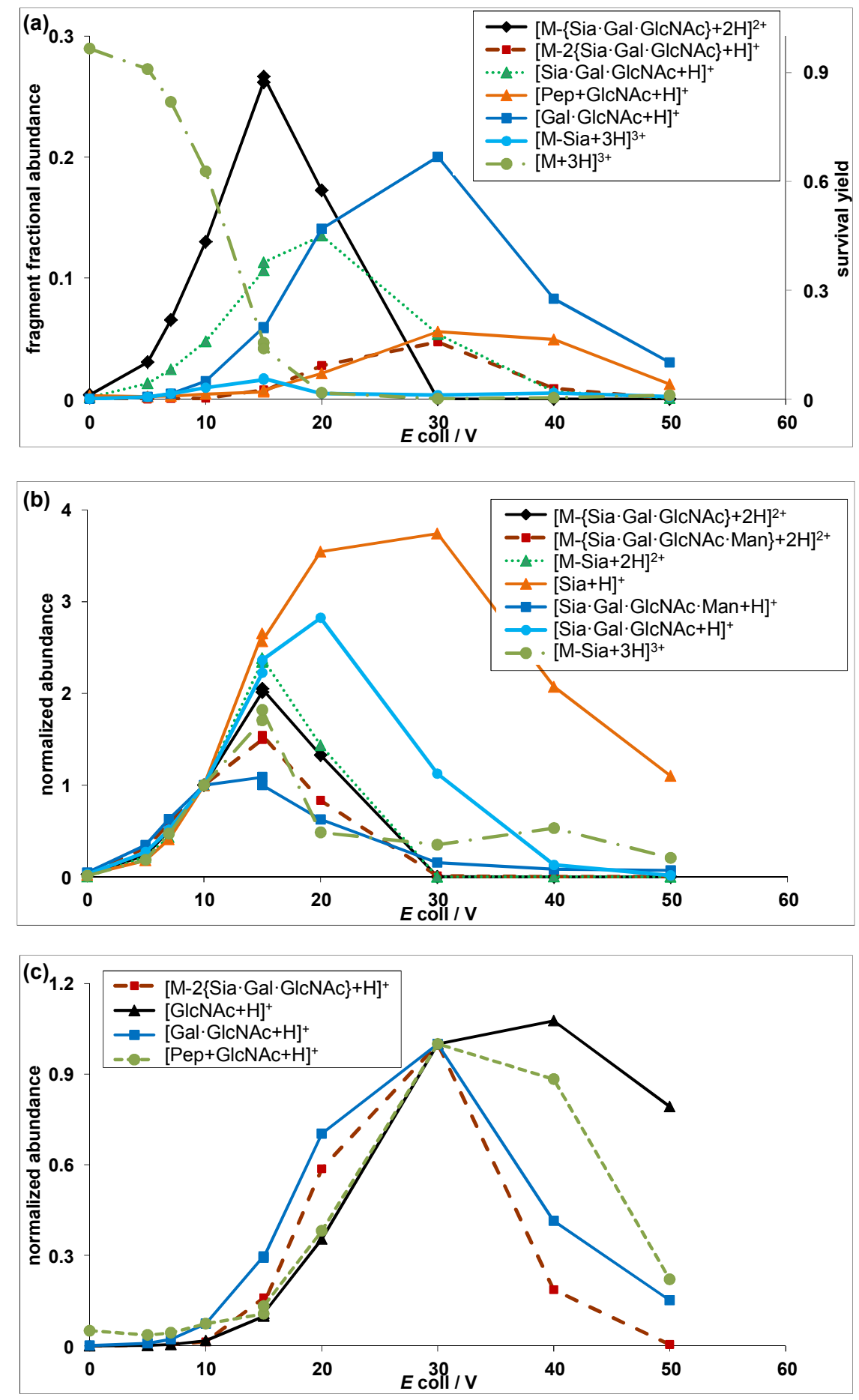
Fig. 4. Survival yield curves of the TriS3.LVPVPITNATLDR glycopeptide; observed for the $2+, 3+$ and $4+$ charge states (2, 3 and 4-times protonated forms).

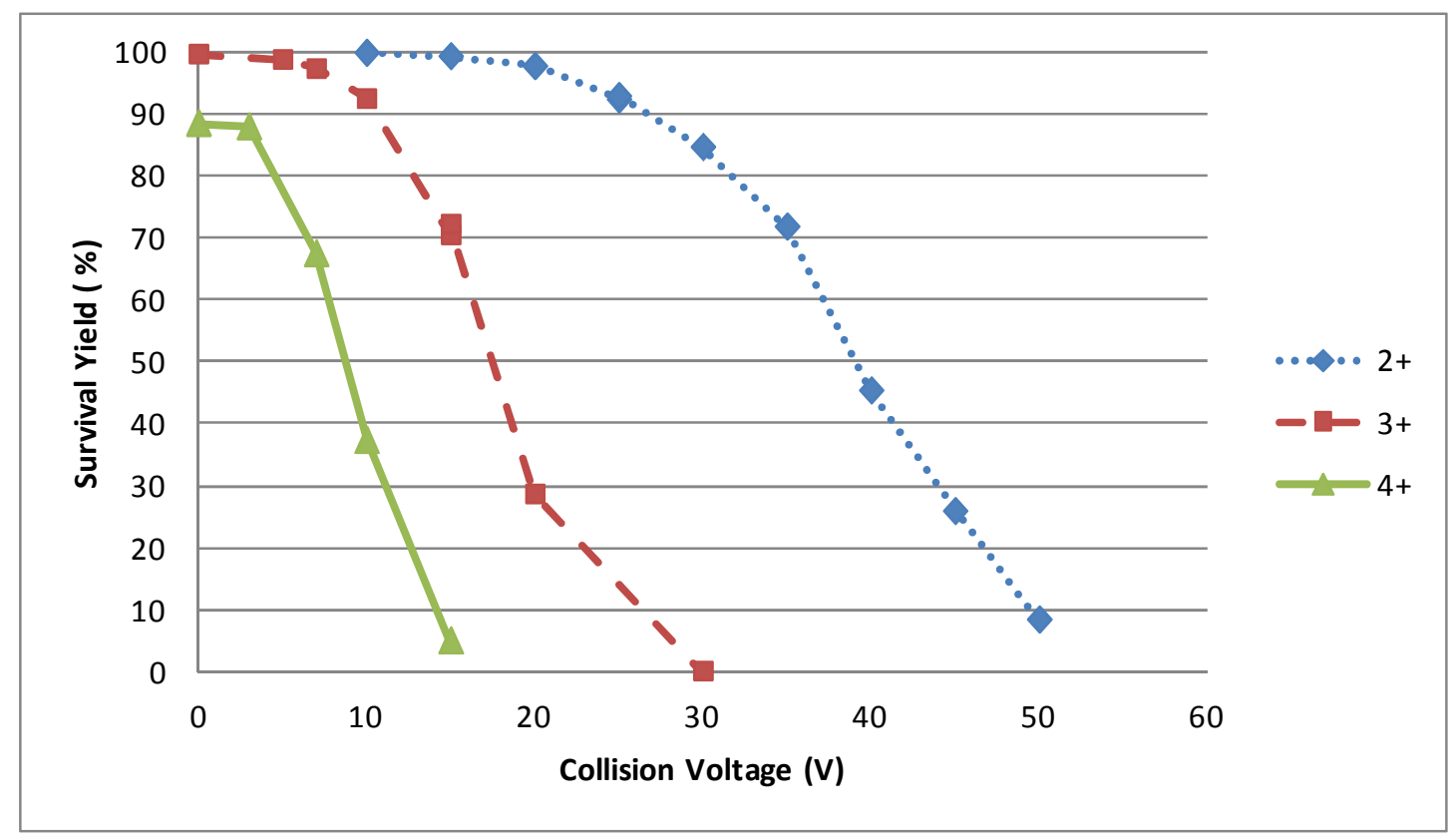


Fig. 5. Tandem mass spectra of doubly, triply and quadruply protonated TriS3.LVPVPITNATLDR glycopeptide, derived from AGP. All spectra correspond to ca. 50\% survival yield; and have been taken at $35 \mathrm{~V}, 15 \mathrm{~V}$ and $7 \mathrm{~V}$ collision voltage. Fragmentation is discussed in detail in the text.

\section{(a) Charge state $=2+$}
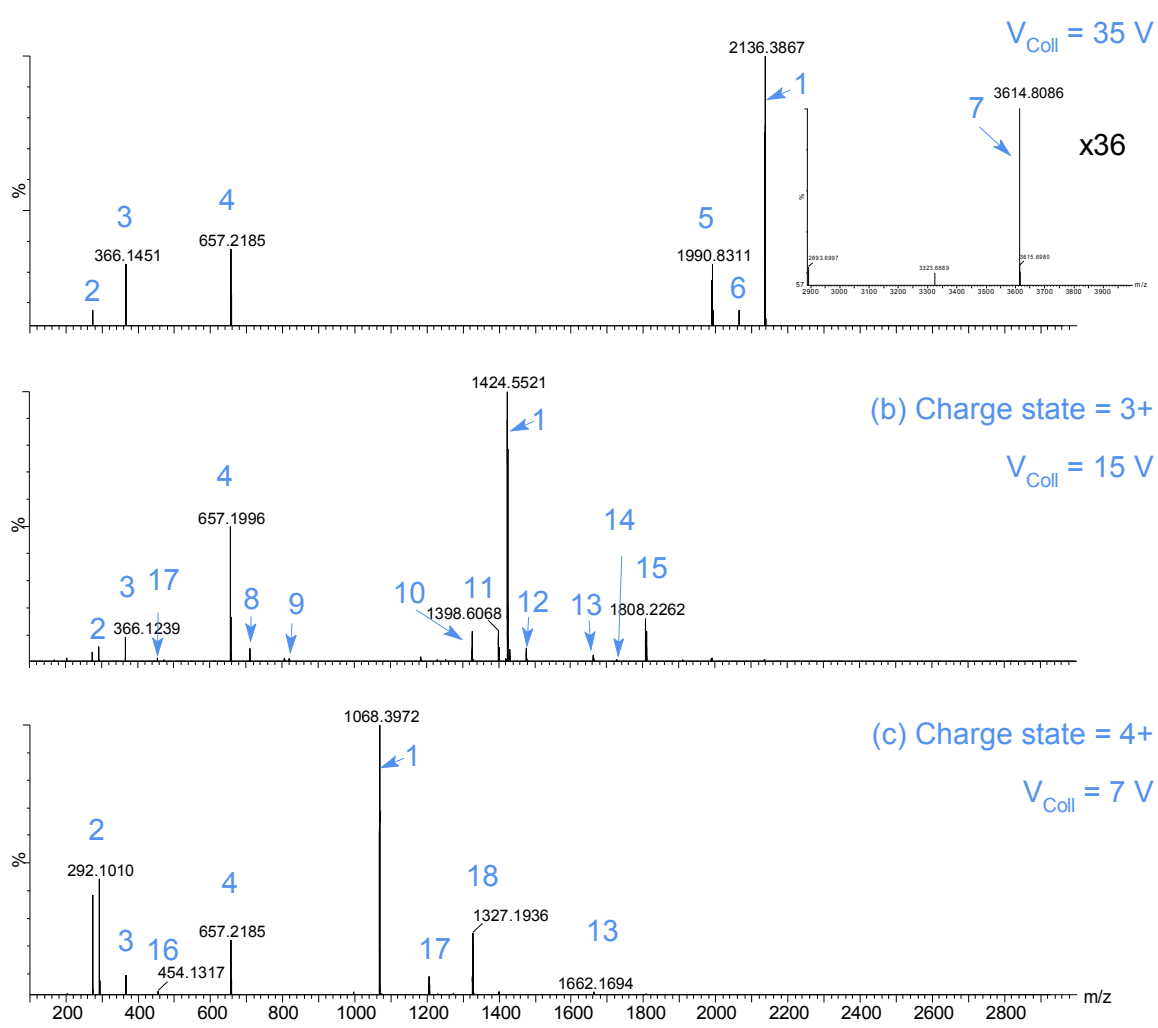

1. $[\mathrm{M}+\mathrm{nH}]^{\mathrm{n}+}$

2. $[\mathrm{Sia}+\mathrm{H}]^{+}$

3. $[\mathrm{Gal} \cdot \mathrm{GlcNAc}+\mathrm{H}]^{+}$

4. $[\mathrm{Sia} \cdot \mathrm{Gal} \cdot \mathrm{GlcNAc}+\mathrm{H}]^{+}$

5. $[\mathrm{M}-\mathrm{Sia}+2 \mathrm{H}]^{2+}$

6. $[\mathrm{M}-\mathrm{Fuc}+2 \mathrm{H}]^{2+}$

7. $[\mathrm{M}-\{\mathrm{Sia} \cdot \mathrm{Gal} \cdot \mathrm{GlcNAc}\}+\mathrm{H}]^{+}$

8. $\left[\mathrm{GlcNAc}_{3} \mathrm{Man}_{3} \mathrm{Gal}_{2}+2 \mathrm{H}\right]^{2+}$

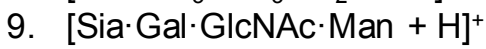

10. $[\mathrm{M}-\mathrm{Sia}+3 \mathrm{H}]^{3+}$

11. [M - \{Sia.Gal·GlcNAc\}$\{\mathrm{Sia} \cdot \mathrm{Gal} \cdot \mathrm{GlcNAc} \cdot \mathrm{Man}\}+2 \mathrm{H}]^{2+}$

12. $\left[\{\mathrm{Sia} \cdot \mathrm{Gal} \cdot \mathrm{GlcNAc}\}_{2} \cdot \mathrm{Man}+\mathrm{H}\right]^{+}$

13. [M- $\{\mathrm{Sia} \cdot \mathrm{Gal} \cdot \mathrm{GlcNAc}\}-\mathrm{Sia}+$ $2 \mathrm{H}]^{2+}$

14. $[\mathrm{M}-\{\mathrm{Sia} \cdot \mathrm{Gal} \cdot \mathrm{GlcNAc} \cdot \mathrm{Man}\}+$ $2 \mathrm{H}]^{2+}$

15. $[\mathrm{M}-\{\mathrm{Sia} \cdot \mathrm{Gal} \cdot \mathrm{GlcNAc}\}+2 \mathrm{H}]^{2+}$

16. $[\mathrm{Sia} \cdot \mathrm{Gal}+\mathrm{H}]^{+}$

17. $[\mathrm{M}-\{\mathrm{Sia} \cdot \mathrm{Gal} \cdot \mathrm{GlcNAc}\}+3 \mathrm{H}]^{3+}$

18. $[\mathrm{M}-\mathrm{Sia}+3 \mathrm{H}]^{3+}$ 
1.

Fig. 6. Low energy CID spectra of selected glycopeptides, containing a fucose residue. See text for discussion. $\quad$ (a) TriS3F1.LVPVPITNATLDR BiS2F1·QQQHLFGSNVTDCSGNFCLFR, (d) N4H3SOF1·EEQYNSTYR

(b)

BiS2F1·NEEYNK,

(c)
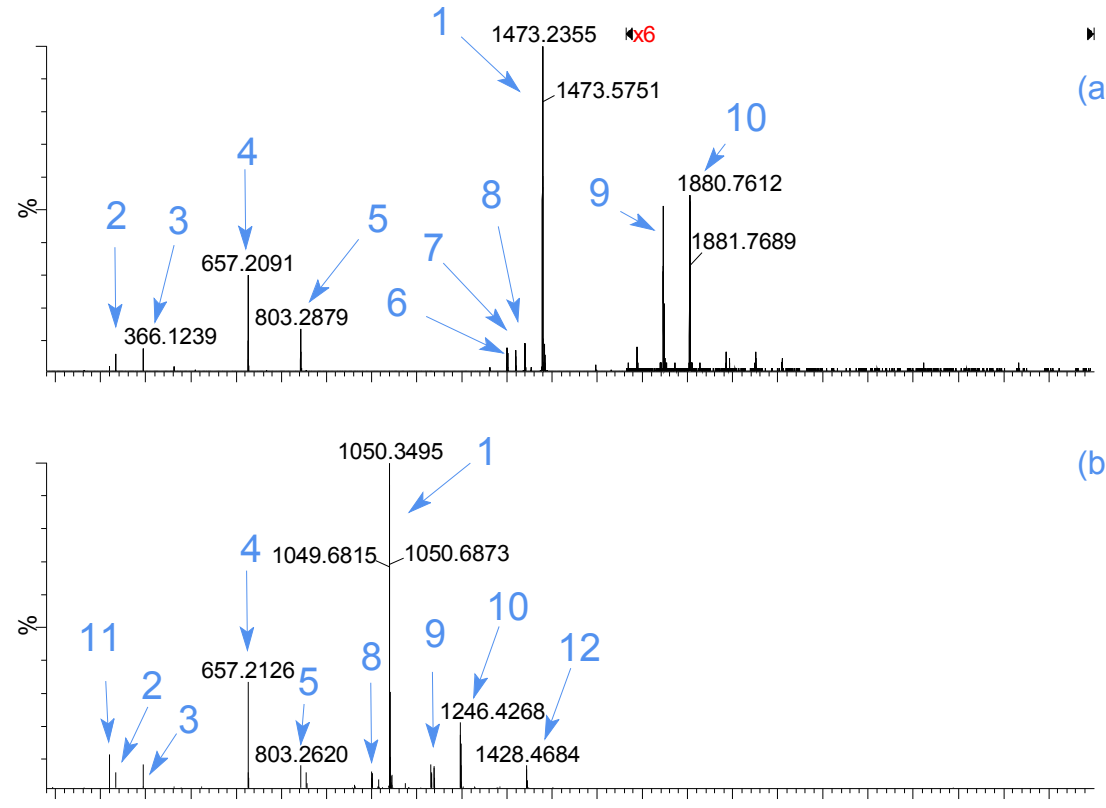

(a)

1. $[\mathrm{M}+3 \mathrm{H}]^{3+}$

2. $[\mathrm{Sia}+\mathrm{H}]^{+}$

3. $[\mathrm{Gal} \cdot \mathrm{GlcNAc}+\mathrm{H}]^{+}$

4. [Sia.Gal.GlcNAc $+\mathrm{H}]^{+}$

5. $[\mathrm{Sia} \cdot \mathrm{Gal} \cdot \mathrm{GlcNAc} \cdot \mathrm{Fuc}+\mathrm{H}]^{+}$

6. $[\mathrm{M}-\mathrm{Sia}+3 \mathrm{H}]^{3+}$

7. $[\mathrm{M}-\{\mathrm{Sia} \cdot \mathrm{Gal} \cdot \mathrm{GlcNAc} \cdot \mathrm{Fuc}\}$ $\{$ Sia.Gal.GlcNAc.Man $\}+2 \mathrm{H}]^{2+}$

b) 8. $[\mathrm{M}-\mathrm{Fuc}+3 \mathrm{H}]^{3+}$

9. $[\mathrm{M}-\{\mathrm{Sia} \cdot \mathrm{Gal} \cdot \mathrm{GlcNAc} \cdot \mathrm{Fuc}\}+2 \mathrm{H}]^{2+}$

10. $[\mathrm{M}-\{\mathrm{Sia} \cdot \mathrm{Gal} \cdot \mathrm{GlcNAc}\}+2 \mathrm{H}]^{2+}$

11. $\left[\mathrm{Sia}-\mathrm{H}_{2} \mathrm{O}+\mathrm{H}\right]^{+}$

12. $[\mathrm{M}-\mathrm{Sia}+2 \mathrm{H}]^{2+}$

13. $[\mathrm{GlcNAc}+\mathrm{H}]^{+}$

14. $[\mathrm{Pep} \cdot \mathrm{GlcNAc}+2 \mathrm{H}]^{2+}$

15. $[\mathrm{Pep} \cdot \mathrm{GlcNAc} \cdot \mathrm{Fuc}+2 \mathrm{H}]^{2+}$

16. $[\mathrm{M}-2 \mathrm{Sia}+3 \mathrm{H}]^{3+}$

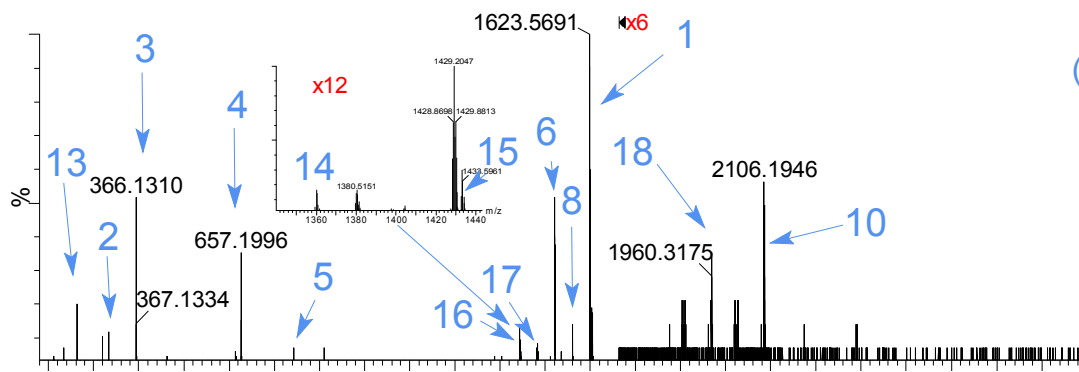

(c) 18. $[\mathrm{M}-\{\mathrm{Sia} \cdot \mathrm{Gal} \cdot \mathrm{GlcNAc}\}-\mathrm{Sia}+2 \mathrm{H}]^{2+}$

19. [GlcNAc. Fuc $+\mathrm{H}]^{+}$

20. [2Man.GlcNAc $+\mathrm{H}]^{+}$

21. $[2 \mathrm{Man} \cdot 2 \mathrm{GlcNAc}+\mathrm{H}]^{+}$

22. $[\mathrm{M}-\{\text { GlcNAc} \cdot M a n \cdot F u c\}+2 \mathrm{H}]^{2+}$

23. $[\mathrm{M}-\{\mathrm{GlcNAc} \cdot \mathrm{Man}\}+2 \mathrm{H}]^{2+}$

24. $[\mathrm{M}-\mathrm{GlcNAc}+2 \mathrm{H}]^{2+}$

25. $[\mathrm{M}-\mathrm{Fuc}+2 \mathrm{H}]^{2+}$

26. $[\mathrm{M}+2 \mathrm{H}]^{2+}$

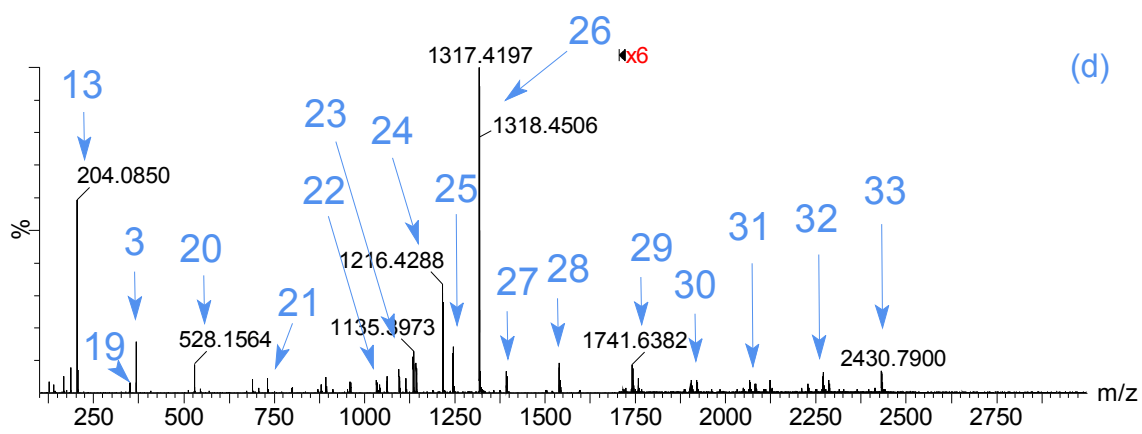

27. $[\mathrm{Pep} \cdot \mathrm{GlcNAc}+\mathrm{H}]^{+}$

28. [Pep $\cdot$ GlcNAc $\cdot F u c+H]$

29. $[\mathrm{M}-2\{\text { GlcNAc} \cdot \operatorname{Man}\}-\mathrm{Man}+\mathrm{H}]^{+}$

30. $[\mathrm{M}-2\{\text { GlcNAc} \cdot \operatorname{Man}\}+\mathrm{H}]^{+}$

31. $[\mathrm{M}-\{\text { GlcNAc} \cdot M a n\}-\mathrm{GlcNAc}+\mathrm{H}]^{+}$

32. $[\mathrm{M}-\{\mathrm{GlcNAc} \cdot \mathrm{Man}\}+\mathrm{H}]^{+}$

33. $[\mathrm{M}-\mathrm{GlcNAc}+\mathrm{H}]^{+}$ 